\title{
Cobertura informativa en Twitter de los diarios españoles de referencia sobre la campaña electoral del $10 \mathrm{~N}$
}

\author{
Informative coverage in Twitter by the Spanish \\ reference newspapers on the $10 \mathrm{~N}$ electoral campaign
}

\author{
Álvaro López-Martín
}

Cómo citar este artículo:

López-Martín, Álvaro (2020). “Cobertura informativa en Twitter de los diarios españoles de referencia sobre la campaña electoral del 10N". Profesional de la información, v. 29, n. 5, e290510.

https://doi.org/10.3145/epi.2020.sep.10

Artículo recibido el 29-11-2019

Aceptación definitiva: 22-04-2020

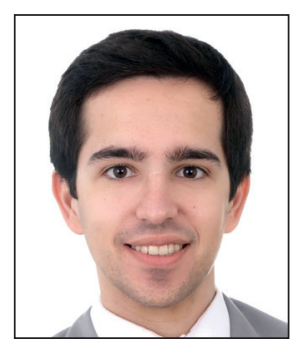

\author{
Álvaro López-Martín \\ https://orcid.org/0000-0001-7871-2137 \\ Universidad de Málaga \\ Facultad de Ciencias de la Comunicación \\ Campus de Teatinos \\ León Tolstoi, 4. 29071 Málaga, España \\ alvarolopezmartin@uma.es
}

\section{Resumen}

Se analiza la cobertura informativa realizada en Twitter por los diarios españoles El país, El mundo, $A B C$ y La vanguardia durante la campaña electoral previa a los comicios generales del 10 de noviembre de 2019. A través del análisis de contenido de todos los tweets publicados por estos medios referidos a las elecciones $(n=1.462)$ entre el viernes 1 y sábado 9 de noviembre, se constata que, aunque existen divergencias en las pautas de producción del mensaje y los rasgos de éste, en todos los diarios este asunto registra una notable incidencia y la cobertura gravita en torno a dos temas principales: el debate electoral del $4 \mathrm{~N}$ y los actos de la campaña. Los resultados reflejan también que, pese a que la mayoría de los tweets presentan un tono neutro, se cuantifican en menor medida mensajes con un posicionamiento claro, existiendo correspondencia con la línea editorial del medio. Se concluye que durante los períodos electorales la agenda mediática gira en torno a esta cuestión, suponiendo aproximadamente una quinta parte del total de las piezas publicadas en Twitter. Los diarios no aprovechan la naturaleza dialógica y las posibilidades que proporciona esta red social para crear comunidad entre sus lectores, limitándose a la difusión de los contenidos de la edición digital.

\section{Palabras clave}

Twitter; Redes sociales; Medios sociales; Prensa de referencia; Diarios; Campañas electorales; Elecciones generales; 10N; Política; Análisis de contenido; España.

\begin{abstract}
This paper analyzes the information coverage by the Spanish newspapers $E l$ país, El mundo, $A B C$, and La vanguardia in Twitter on the electoral campaign prior to the 10 November 2019 general elections. A content analysis of all tweets published by these media regarding the elections $(n=1,462)$ between Friday 1 and Saturday 9 November reveals that, although there are differences in the production patterns and features of the messages, the subject received notable attention in all the newspapers. The coverage gravitated around two main themes: the $4 \mathrm{~N}$ electoral debate, and campaign events. The results reveal that, although most of the tweets had a neutral tone, messages with clear positioning corresponding to the editorial line of the newspaper were also published, albeit to a lesser extent. During the electoral period, the media agenda revolved around this issue, accounting for around one-fifth of all pieces published on Twitter. Newspapers do not take advantage of the dialogic nature or possibilities offered by this social network to create a community among their readers, limiting themselves to the dissemination of content from their digital edition.
\end{abstract}

\section{Keywords}

Twitter; Social networks; Social media; Reference press; Newspapers; Electoral campaigns; General election; 10N; Politics; Content analysis; Spain. 


\section{Introducción}

La transformación del panorama comunicativo experimentada en los últimos años está estrechamente vinculada con la implantación de las nuevas tecnologías. En este contexto emergente los medios sociales han contribuido a consolidar la Red como un innovador espacio para la socialización y mantenerse informado, un entorno al que además de los ciudadanos también se han sumado organizaciones, administraciones públicas y empresas informativas, entre otros. Facebook, Instagram y, especialmente, Twitter son las redes sociales más destacadas para la diseminación de información (Alonso-Muñoz; Miquel-Segarra; Casero-Ripollés, 2016), empleando los medios de comunicación estas vías como altavoces para distribuir y promocionar sus contenidos.

La red de microblogging Twitter cuenta con 139 millones de usuarios activos diariamente en todo el mundo (Moreno, 2019). Según Chung y Buhalis (2008), la búsqueda de información y la creación de comunidades y el entretenimiento son las principales motivaciones que llevan a los internautas a emplear este medio social, el cual se constituye como la red que concentra un mayor número de personas interesadas en la actualidad (Pujol, 2019). Es preciso mencionar un informe de Pew Research Center (2018) en el que se señala que el $61 \%$ de la población española se informa a través de las redes sociales. Estos datos, junto a la influencia que ejercen los medios en la construcción de la

\section{El 61\% de la población española se infor-} ma a través de las redes sociales opinión pública (Guemureman et al., 2011), suscitan un notable interés por conocer la cobertura que llevan a cabo los medios de comunicación sobre hechos de gran relieve e importancia, como los procesos electorales.

Los trabajos previos relacionados con el uso de Twitter en períodos electorales han puesto el foco principalmente en el análisis de su utilización por parte de candidatos y partidos políticos (Enli; Skogerbo, 2013; Quevedo-Redondo; Portalés-Oliva; Berrocal-Gonzalo, 2016; Gómez-Calderón; Roses; Paniagua-Rojano, 2017), pero no abundan las investigaciones que aborden la cobertura realizada por los medios sobre estos procesos a través de dicho canal. Por ello este trabajo pretende paliar, al menos de manera parcial, este vacío en la bibliografía científica y complementar con sus hallazgos las investigaciones previas.

\subsection{Incidencia de Twitter en el ecosistema político y periodístico}

Con el surgimiento de internet y las posibilidades que brinda este fenómeno, el panorama comunicativo ha experimentado una notable transformación en todas sus facetas. Una de ellas es el de la comunicación política, siendo hoy condición sine qua non el uso de las redes sociales en las estrategias comunicativas de las campañas electorales (Rodríguez-Andrés; Ureña-Uceda, 2011; Alonso-Muñoz; Miquel-Segarra; Casero-Ripollés, 2016), constituyéndose Twitter como la red social

"que más relieve ha conseguido entre políticos y periodistas" (García-Ortega; Zugasti-Azagra, 2014, p. 300).

Tras la cibercampaña llevada a cabo por Barack Obama en las elecciones a la presidencia de Estados Unidos de 2008 (Gainous; Wagner, 2014; De-Aguilera; Casero-Ripollés, 2018), en España las potencialidades de Twitter comenzaron a experimentarse en 2009 en los comicios autonómicos de Galicia y País Vasco (Criado; Martínez-Fuentes; Silván, 2013; Alonso-Muñoz; Miquel-Segarra; Casero-Ripollés, 2016), convirtiéndose desde entonces su uso en una práctica habitual de los políticos con el propósito de establecer un vínculo directo con los votantes (Gómez-Calderón; Roses; Paniagua-Rojano, 2017). Estos últimos años la influencia ejercida por las redes sociales ha quedado demostrada en la organización y éxito de diversos procesos sociopolíticos, en los que estos medios desempeñaron un papel destacado: movimiento 15-M en España (Micó; Casero-Ripollés, 2014) u Occupy Wall Street de Estados Unidos (Castillo-Esparcia; García-Ponce; Smolak-Lozano, 2013).

Los medios de comunicación han reaccionado a este fenómeno y, al igual que ocurrió con las ediciones digitales, las empresas informativas se han adaptado a este emergente ecosistema abriendo sus perfiles oficiales en redes sociales (Zamarra, 2015), lo que ha favorecido la obtención de lectores y por tanto un mayor tráfico en sus respectivas webs. Salgado y Zamarra-López sostienen al respecto que

"ya no es posible desligar a los medios de las redes sociales. Algo que han aprovechado para estar presentes e intentar encontrar ahí un público dinámico" (Salgado; Zamarra-López, 2013, p. 61).

Diversos autores inciden en el potencial de las redes sociales para un óptimo posicionamiento de los medios en el mercado informativo (Ju; Jeong; Chyi, 2014; Tandoc; Vos, 2016). En términos similares se expresan González-Molina y Ramos-del-Cano, quienes consideran que los medios sociales son recursos fundamentales para lograr

"una comunicación continua, basada en la sencillez y en la interactividad [...] además de un posicionamiento rápido y eficaz en el mercado" (González-Molina; Ramos-del-Cano, 2014, p. 38),

lo que motiva un cambio en la comunicación estratégica de los medios con sus audiencias. Otros autores como Hong (2012) ven en el auge de las redes sociales una de las principales causas del incremento del número de lectores de los medios, dado el impacto y alcance que posibilita la distribución de las noticias por estas vías. 
Esta red social da pie además a entablar una conversación o interacción con el propio medio y a la creación de una comunidad de internautas que se sientan identificados con la cabecera (Fernández-Gómez; Martín-Quevedo, 2018). Así, los retweets, "me gusta" o comentarios realizados en las publicaciones son indicadores para conocer, de manera parcial, la fidelidad o el compromiso de la audiencia con el medio (Huertas; Setó-Pàmies; Míguez-González, 2015). Esta novedosa conexión medios-audiencias puede suponer una oportunidad para que los lectores recuperen la confianza en las empresas informativas, ofreciendo una imagen más abierta y transparente. De hecho, la proyección de marca, el aumento del tráfico de los contenidos y la búsqueda de la fidelidad del público constituyen los principales propósitos de los medios a la hora de emplear las redes sociales (García-De-Torres et al., 2011).

Si bien Twitter tiene múltiples utilidades, la difusión de contenidos periodísticos es la más empleada por los medios de comunicación, dadas las atractivas posibilidades que presenta (Cobos, 2015). Túñez-López destaca que esta red social posibilita el envío de titulares sin que el mensaje requiera una nueva elaboración, aunque apostilla que

"las principales cabeceras personalizan su envío buscando proximidad y empatía con un lenguaje coloquial y segunda persona en el trato al usuario" (Túñez-López, 2012, p. 231).

La inmediatez y universalidad como cualidades asociadas a sus mensajes han motivado que casi la totalidad de las empresas informativas empleen este canal para realizar coberturas o seguimientos de acontecimientos informativos de relevancia. Precisamente la inmediatez -rasgo vinculado también a la web 2.0- es una de las cuestiones que mayor incidencia ha ejercido en la mutación experimentada por el periodismo. Esto hace que en palabras

Inmediatez y universalidad son dos de las características de los tweets de Paz-García y Spinosa (2014, p. 136), Twitter se haya convertido en "un medio viral que facilita la rápida circulación y multiplicación de los mensajes", constituyéndose como la principal fuente informativa (Nadal-Martínez, 2013).

Aunque el uso que hacen de las redes sociales los internautas no está profesionalizado, de manera frecuente emplean estos espacios para la producción de información (Domingo; Masip; Costera-Meijer, 2014; Linares-Lanzman; Pérez-Altable, 2015), convirtiéndose en emisores y productores de contenidos, ejerciendo de fuentes de información para los propios periodistas (Micó; Casero-Ripollés, 2014; Sánchez-García; Campos-Domínguez; Berrocal-Gonzalo, 2015). Bruns y Burgess (2012) estiman por ello que Twitter es el mejor ejemplo de tecnología en la que confluyen el intercambio de información, la producción de contenidos y la convergencia de los medios sociales. Es preciso destacar la proliferación y popularización de los smartphones, lo que ha facilitado que las redes sociales "estén" siempre en el lugar de la noticia y los internautas generen contenidos sobre el hecho in situ e instantáneamente (Linares-Lanzman; Pérez-Altable, 2015). Todo ello ha derivado en una transformación de las redacciones, así como de las rutinas profesionales (Artwick, 2013; Palomo; Palau-Sampio, 2016), suponiendo un novedoso campo para el ejercicio periodístico.

\section{Objetivos}

El objetivo fundamental de esta investigación es explorar la cobertura informativa realizada por los principales diarios de referencia españoles en sus perfiles de Twitter sobre la campaña electoral de los comicios generales celebrados el 10 de noviembre de 2019. Esto ayudará a dilucidar el uso que hacen de esta red social las cabeceras de referencia para informar sobre uno de los acontecimientos o períodos que mayor atención genera por parte de los medios de comunicación.

En aras de una mayor concreción se han establecido cuatro objetivos subsidiarios. En primer lugar, se pretende calibrar la incidencia que tiene este tema respecto al total de los tweets registrados (01), un dato que ayudará a conocer de manera cuantitativa la atención que cada medio otorga a la campaña electoral. Otro de los aspectos que interesa es examinar los rasgos del tweet, con el fin de establecer un patrón de utilización de esta red social, analizando la manera en la que se presentan estas cuestiones ante la audiencia y la interacción que logran -a través de los retweets, favoritos y comentarios registrados- con dichos mensajes los medios de comunicación (02). También se persigue identificar los macrotemas que abordan predominantemente los medios durante la campaña electoral (O3). Finalmente, dado el alto paralelismo político que caracteriza al sistema de medios español y la notable influencia que ejercen los medios de comunicación en la construcción de la opinión pública, en esta investigación se pretende conocer si la cobertura realizada guarda relación con la línea editorial de la cabecera (O4).

\section{Metodología}

Para la consecución de los objetivos de la investigación se ha empleado un método basado en el análisis de contenido mixto. En cuanto al análisis cuantitativo, la elección de esta técnica se debe a su idoneidad para el tratamiento de un elevado volumen de información o mensajes periodísticos (Krippendorff, 1990), así como a la alta fiabilidad y a la flexibilidad instrumental que proporciona (Wimmer; Dominick, 1996; Igartua-Perosanz, 2006; Barredo, 2015). Para el análisis cualitativo, de acuerdo con investigaciones previas sobre esta red social, se ha recurrido al análisis semántico y a la búsqueda de palabras clave (Tumasjan et al., 2010; Said-Hung; Arcila-Calderón, 2011). Esto permitirá estudiar el tono del tweet y se pondrá el foco principalmente en el empleo de los adjetivos calificativos y el carácter del mensaje -positivo o negativo-.

Se pretende conocer la cobertura informativa realizada por los diarios españoles de referencia en Twitter durante la campaña electoral del $10 \mathrm{~N}$ 
En este trabajo se seleccionaron todos los mensajes publicados en los perfiles oficiales de Twitter de los diarios El país, El mundo, $A B C$ y La vanguardia $(\mathrm{N}=6.553)$ entre el viernes 1 de noviembre de 2019 -primer día de campaña electoral- y el sábado 9 del mismo mes - día posterior al cierre de la campaña-, poniéndose el foco de análisis sobre los tweets referidos a las elecciones generales $(n=1.462)$. La muestra quedó constituida de la siguiente manera:

- 491 de El país (@el_pais)

- 302 de El mundo (@elmundoes)

- 367 de $A B C$ (@abc_es)

- 302 de La vanguardia (@LaVanguardia).

La elección de estos medios responde a criterios cuantitativos, dado que son las cuatro cabeceras nacionales generalistas con mayor número de seguidores en Twitter:

- 7,14 millones El país

- 3,41 El mundo

- 1,76 ABC

- 1,08 La vanguardia.

La ficha de análisis se estructuró en tres bloques con diversas variables:

a) Información de registro: datos para la identificación de la pieza para su posterior disección.

b) Contenido: se contemplan las variables sobre los elementos lingüísticos y paralingüísticos que conforman el mensaje, con las que se exploran las propiedades formales de los tweets publicados.

b.1) Tipo: se distingue entre tweets básicos - los generados por el propio medio- y retweets.

b.2) Temática: los hechos sobre los que se pone el foco en los mensajes. Se han distinguido ocho categorías:

- actos de campaña;

- polémicas sobre partidos políticos o sus dirigentes;

- debates: en este bloque, a su vez se distingue entre el debate a cinco, debate a siete, debate femenino y otros debates;

- análisis y opinión;

- entrevistas;

- elecciones del 10N: aquí se incluyen las piezas relacionadas con las encuestas, el voto por correo y el conflicto y manifestaciones de Cataluña, entre otros;

- pactos-acuerdos;

- generalidades.

b.3) Elementos visuales: se identifican los elementos paralingüísticos e icónicos (fotografías, vídeos, gifs...) que se emplean en el tweet. Maciá y Santonja (2016) apuntan que los seguidores se fijan principalmente en las imágenes, antes que en el texto, lo que hace que el análisis de este apartado cobre relevancia.

b.4) Hipertextualidad: se pone el foco en los hipertextos incluidos en los mensajes, distinguiendo en qué medida conducen a noticias del mismo diario o si, por el contrario, comparten publicaciones de otro medio.

b.5) Uso de hashtags o etiquetas: el uso de estos recursos, de acuerdo con López-Meri (2015), ayuda a dar mayor difusión a los contenidos. En este apartado nos ocupamos del empleo y frecuencia de los hashtags registrados en los mensajes.

b.6) Elaboración textual: se examina lo referido a la narrativa, distinguiendo entre los mensajes que son elaborados y los que reproducen los titulares de la edición digital.

c) Interacción: se incluye una serie de variables sobre la interacción o respuesta que los medios obtienen de sus audiencias, como los retweets, favoritos y comentarios registrados en los tweets sobre la campaña electoral.

\section{Resultados}

\subsection{Incidencia}

Los resultados del análisis muestran en líneas generales una notable incidencia de los tweets referidos a las elecciones respecto al total de los mensajes publicados por las cuatro cabeceras. Atendiendo a los datos globales de la campaña electoral, como se aprecia en la tabla 1 la presencia de este hecho supone entre el 19,38 (La vanguardia) y el 24,01\% (EI país) del total. El diario del grupo Prisa es el que registra más publicaciones sobre la campaña electoral (491). Frente a esto, La vanguardia y El mundo son los medios que menos mensajes publican sobre este asunto, aunque la incidencia que ejerce en esta última cabecera es superior.

Aunque no se aprecia un patrón común en los cuatro medios, sí se observa una mayor producción durante las jornadas del lunes 4 y martes 5 en tres de los diarios, coincidiendo estas fechas con la celebración del debate televisivo de los líderes políticos (lunes 4) y el día pos-
Los días de mayor producción de mensajes coinciden con la celebración del debate electoral 
terior, en el cual los tweets sobre ello fueron abundantes. El día del debate electoral la incidencia registrada en El país alcanza el $46,17 \%$, seguido del 34,78 y $25,63 \%$ en $E$ I mundo y $A B C$ respectivamente. En La vanguardia dicho día la incidencia es moderada, lo que confronta con los datos registrados el martes 5, en el cual los tweets referidos a la campaña representan casi la mitad del total $(48,25 \%)$. En el resto de los medios ocurre lo contrario y el porcentaje de tweets publicados el martes 5 de noviembre desciende.

El viernes 8 es otra jornada en la que esta materia tiene una destacada incidencia en los cuatro medios, registrándose en todos ellos datos muy similares, en número de publicaciones y en porcentaje. Durante el fin de semana de la campaña electoral, así como el sábado 9 -la denominada jornada de reflexión-, se detecta una caída del número de tweets publicados. Una tendencia cuya explicación puede apoyarse en la propia rutina de los medios, cuyo ritmo desciende en sábados y domingos. En estos días la campaña electoral centra menos atención en El país y La vanguardia, cuya incidencia respecto al total de los mensajes publicados el domingo 3 no supera el 13,3 y 6,83\% respectivamente, mientras que en $E$ I mundo y $A B C$ la atención prestada a las cuestiones políticas es similar o superior a la registrada otros días.

Tabla 1. Incidencia de los tweets relativos a la campaña electoral

\begin{tabular}{|l|c|c|c|}
\hline \multicolumn{1}{|c|}{ Día } & El país & El mundo & ABC vanguardia \\
\hline Viernes 1 & $32(17,20 \%)$ & $23(18,11 \%)$ & $40(23,95 \%)$ \\
\hline Sábado 2 & $28(13,65 \%)$ & $26(21,31 \%)$ & $33(21,29 \%)$ \\
\hline Domingo 3 & $29(13,30 \%)$ & $20(18,18 \%)$ & $36(22,22 \%)$ \\
\hline Lunes 4 & $169(46,17 \%)$ & $72(34,78 \%)$ & $61(25,63 \%)$ \\
\hline Martes 5 & $62(26,38 \%)$ & $44(27,16 \%)$ & $47(23,03 \%)$ \\
\hline Miércoles 6 & $33(17,01 \%)$ & $28(16,47 \%)$ & $39(18,93 \%)$ \\
\hline Jueves 7 & $55(22,26 \%)$ & $27(16,26 \%)$ & $41(21,13 \%)$ \\
\hline Viernes 8 & $50(23,69 \%)$ & $41(25,78 \%)$ & $48(25,53 \%)$ \\
\hline Sábado 9 & $33(18,03 \%)$ & $21(23,33 \%)$ & $44(18,33 \%)$ \\
\hline Total campaña: & $491(4,01 \%)$ & $302(23,00 \%)$ & $22(17,88 \%)$ \\
\hline
\end{tabular}

\subsection{Tipo}

Se aprecia que la mayoría de los mensajes -casi la totalidad en $A B C$ y La vanguardia- responden a la categoría de tweets básicos, es decir, los generados por el propio medio. En menor medida las cabeceras estudiadas comparten mensajes publicados por otras cuentas, aunque esta acción sólo alcanza valores notables en El país y El mundo, en los que el 19 y $17 \%$ respectivamente de los mensajes sobre la campaña electoral son procedentes de otros perfiles. Si bien estas publicaciones no son generadas por la cuenta principal del medio, sí proceden de perfiles subsidiarios o vinculados a la cabecera,como @elpais_foto, @elpaissemanal, @elpais_opinion, @elpaisinenglish, @EIMundoOpinion, @EIMundoEspana, @Cronica_EIMundo o @TelevisionLV, entre otros perfiles, así como de periodistas de la propia cabecera. Por otra parte, $A B C$ comparte un total de seis tweets procedentes de la cadena COPE, radio con la que el grupo Vocento-propietario de la cabecera- tiene acuerdos empresariales. También se registran dos retweets realizados a políticos:

- La vanguardia retweetea un mensaje de Pedro Sánchez, candidato del PSOE, en el que publica el enlace a una entrevista realizada para dicho medio;

- $A B C$ comparte un tweet generado por el dirigente del Partido Popular Alberto Núñez Feijóo en el que figura un artículo publicado en ese diario con una defensa a su persona.

En ambos casos los retweets pueden formar parte de la autopromoción obligada que tienen que practicar los medios, primando razones comerciales respecto a las ideológicas. No obstante, en $A B C$ sí hay mayores evidencias de la orientación ideológica del diario, ya que el artículo y el tweet de Feijóo sirven para ensalzar la figura del político.

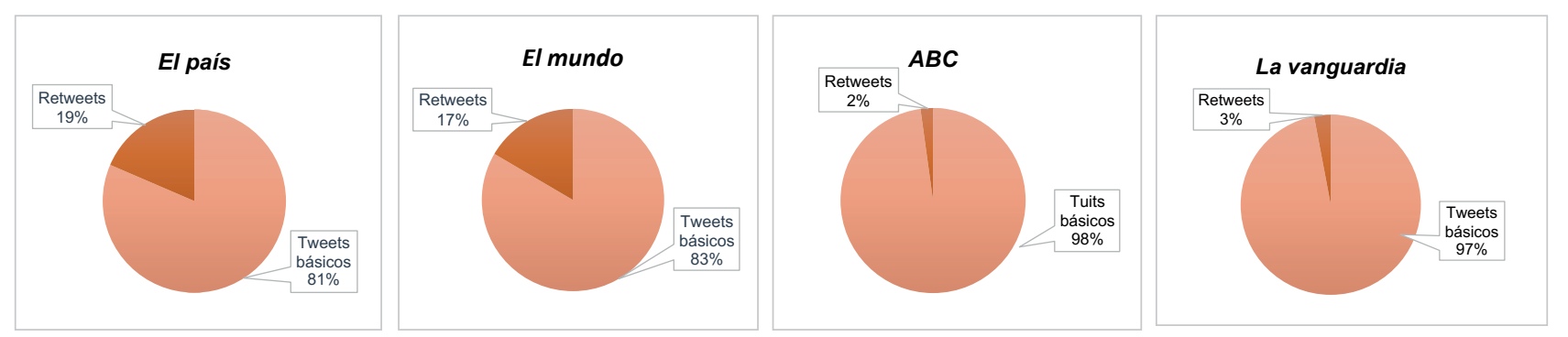

Gráfico 1. Tipo de los tweets publicados

\subsection{Interacción}

Destaca el elevado grado de interacción registrado en El país y El mundo, cuyos promedios de retweets, favoritos y comentarios es notablemente superior al de $A B C$ y La vanguardia, aunque es preciso apostillar que estos dos últimos medios son los que cuentan con un menor número de seguidores en esta red social. 
Se aprecia que el público opta principalmente por la posibilidad de indicar como favorito o "me gusta" el contenido, más que por compartir (retweet) el mensaje para mostrar su aceptación y en menor medida, emiten comentarios $u$ opiniones al respecto de lo publicado. El número de favoritos registrados es casi el doble que el de retweets, un dato del que se infiere que los internautas prefieren evitar compartir contenido político en sus perfiles. En cuanto al análisis pormenorizado destaca El mundo, dado que si bien cuenta con menos de la mitad de seguidores que El país, sus resultados de interacción o feedback logrado -a excepción de los comentarios generados- con la audiencia es superior al de la cabecera de Prisa, registrándose un promedio de 103,49 favoritos y 54,81 retweets por mensaje emitido. El poco empleo que hace este medio de los elementos gráficos y multimedia (fotografías, videos, hashtags...) en sus tweets (tabla 3 ) pone más en valor las cifras de interacción obtenidas por esta cabecera.

Otro dato de interés es el análisis de las cuestiones que mayor reacción generan entre la audiencia, detectándose que, en líneas generales, los tweets referidos a polémicas vinculadas con los partidos políticos y/o sus dirigentes (Caso Gürtel, ERE de Andalucía, firma de proyectos por parte de Rocío Monasterio, etc.), así como los mensajes sobre Santiago Abascal (Vox) y, especialmente, Pablo Iglesias (Unidas Podemos) son los que registran un promedio de retweets, favoritos y comentarios más elevado.

Tabla 2. Interacción lograda con los tweets sobre la campaña electoral

\begin{tabular}{|c|c|c|c|c|c|c|}
\hline \multirow[b]{2}{*}{ Medio } & \multicolumn{2}{|c|}{ Retweets } & \multicolumn{2}{|c|}{ Favoritos } & \multicolumn{2}{|c|}{ Comentarios } \\
\hline & Total & Promedio & Total & Promedio & Total & Promedio \\
\hline El país & 23.241 & 47,33 & 42.583 & 86,77 & 14.006 & 28,52 \\
\hline El mundo & 16.554 & 54,81 & 31.256 & 103,49 & 7.328 & 24,26 \\
\hline$A B C$ & 9.561 & 26,05 & 15.504 & 42,24 & 3.302 & 8,99 \\
\hline La vanguardia & 4.473 & 14,81 & 8.318 & 27,54 & 3.442 & 11,39 \\
\hline
\end{tabular}

\subsection{Características del mensaje}

El repertorio de elementos gráficos empleados en los mensajes se demuestra en la tabla 3 que es limitado, basándose principalmente en la inclusión de fotografías y vídeos. Si bien la incidencia de estos recursos fluctúa de unas cabeceras a otras, en ninguna de ellas alcanza valores que denoten una notable presencia en los tweets. Sí que destaca El país, por su variedad -en menor medida también utiliza gifs, lo cual dota de dinamismo al mensaje, siendo el único diario en el que se registra este elemento-y su incidencia, predominando el empleo de vídeos en el $26,7 \%$ de los tweets publicados. El uso que hacen del vídeo El mundo y La vanguardia en esta red social es moderada y en $A B C$ es (casi) nula. Con mayor frecuencia recurre este último medio a la inclusión de fotografías en sus mensajes de Twitter, siendo junto con El país los dos diarios en los que la imagen tiene mayor incidencia, unos datos que contrastan con las discretas cifras registradas en los otros dos medios.

En cuanto a la narrativa de los mensajes, hay divergencias apreciables, observándose principalmente dos patrones de actuación. Mientras que $A B C$ y $E$ I mundo ( 71,66 y 49,33\% respectivamente) optan en gran medida por reproducir el titular de la noticia, los tweets de El país y La vanguardia son mayoritariamente elaborados, detallándose en el contenido de los mismos los aspectos con mayor interés o las claves del hecho informativo, aportándole al lector una breve exposición general de la noticia. Mayor similitud se detecta en la función de los tweets. La mayoría contiene enlaces a noticias del mismo medio, de lo cual se infiere que se pretende promocionar y promover los contenidos de los respectivos diarios. Tan sólo en $A B C$ se registran enlaces a otro medio -aun así, su incidencia respecto al total es limitada-, todos ellos dirigidos a la web de la cadena COPE.

En cuanto al uso de etiquetas, ninguno de los medios recurre en abundancia al empleo de hashtags, sólo despuntando en este apartado El país, el cual dispone de este método de posicionamiento en el 41,55\% de las unidades de registro. En el análisis se detectan varias etiquetas vinculadas a las elecciones generales o la campaña electoral, de las cuales tan sólo dos de ellas son empleadas en los cuatro medios (\#DebateElectoral y \#10N) y una en tres cabeceras (\#Elecciones10N). También se constata un extenso repertorio de etiquetas asociadas a los debates celebrados, siendo La vanguardia la que mayor diversidad de hashtags emplea: \#UltimaOportunidadL6 y \#EIDebate4N (EP y LV), \#debate7rtve (EP), \#Debatea7RTVE $(A B C)$, \#Debate10N, \#debatv3, \#DebatTV3 y \#Debate7N (LV). Esta disparidad de hashtags sobre los debates confronta con el consenso que se registra a la hora de emplear etiquetas para referirse a la campaña o las propias elecciones, para lo que utilizan casi de maEl país y La vanguardia optan por tweets elaborados, mientras que $A B C$ y El mundo reproducen el titular de la noticia 
Tabla 3. Elementos empleados en la elaboración del tweet

\begin{tabular}{|c|c|c|c|c|}
\hline Elementos & Elpaís & El mundo & $A B C$ & La vanguardia \\
\hline Tweets con foto & $61(12,42 \%)$ & $7(2,32 \%)$ & $54(14,71 \%)$ & $5(1,65 \%)$ \\
\hline Tweets con video & $131(26,70 \%)$ & $41(13,57 \%)$ & $1(0,27 \%)$ & $37(12,25 \%)$ \\
\hline Tweets con gifs & $5(1,02 \%)$ & - & - & - \\
\hline Tweets con enlaces al medio & $479(97,55 \%)$ & $299(99,00 \%)$ & $360(98,09 \%)$ & $264(87,42 \%)$ \\
\hline Tweets copiados del titular & $43(8,75 \%)$ & $149(49,33 \%)$ & $263(71,66 \%)$ & $32(10,59 \%)$ \\
\hline Enlaces a otro medio & - & - & $6(1,63 \%)$ & - \\
\hline Tweets con hashtags & $204(41,55 \%)$ & $52(17,22 \%)$ & $76(20,71 \%)$ & $67(22,18 \%)$ \\
\hline \multicolumn{5}{|l|}{ Principales hashtags empleados } \\
\hline \#DebateElectoral & 149 & 45 & 51 & 34 \\
\hline$\# 10 \mathrm{~N}$ & 101 & 6 & 13 & 24 \\
\hline \#Elecciones10N & 1 & - & 13 & 27 \\
\hline
\end{tabular}

\subsection{Macrotemas}

Los debates políticos -principalmente el debate a cinco celebrado el 4 de noviembre- y los actos de la campaña electoral son los dos macrotemas que más atención concitan entre los medios. El tema con mayor incidencia en El país y EI mundo es el mencionado debate a cinco, seguido de los actos de campaña. Inverso orden en cuanto a la presencia de estos asuntos se registra en $A B C$ y $L a$ vanguardia, en los que la campaña electoral se aborda en el 24,52 y $34,44 \%$ de los tweets respectivamente. Aunque a lo largo del período de análisis se han producido varios debates además del mencionado (debate a siete, debate de mujeres y de TV3, entre otros), la incidencia que han tenido estos otros en las cuatro cabeceras es parca, registrándose vaLos debates electorales y los actos de la campaña son los dos macrotemas que más atención concitan entre los medios lores muy discretos.

En menor medida destacan otros dos macrotemas con notable presencia:

- las piezas opinativas y de análisis;

- la publicación de noticias sobre polémicas vinculadas con algún partido político y/o sus dirigentes.

Frente a esto, las entrevistas a líderes políticos, los posibles pactos y acuerdos, las elecciones del $10 \mathrm{~N}$-en sentido amplio- y los hechos relacionados con la organización y logística de las mismas tienen una presencia reducida en las unidades de análisis.

Tabla 4. Macrotemas abordados en los tweets

\begin{tabular}{|c|c|c|c|c|}
\hline Temática & El país & El mundo & $A B C$ & La vanguardia \\
\hline Actos de campaña & $71(14,46 \%)$ & $75(24,83 \%)$ & $90(24,52 \%)$ & $104(34,44 \%)$ \\
\hline Polémicas & $30(6,11 \%)$ & 27 (8,94\%) & $25(6,81 \%)$ & $18(5,96 \%)$ \\
\hline \multicolumn{5}{|l|}{ Debates } \\
\hline Debate a 5 & 191 (38,90\%) & $82(27,15 \%)$ & $78(21,25 \%)$ & $62(20,53 \%)$ \\
\hline Debate a 7 & $20(4,07 \%)$ & $3(1,00 \%)$ & $16(4,36 \%)$ & $1(0,33 \%)$ \\
\hline Debate femenino & $23(4,68 \%)$ & $7(2,32 \%)$ & $3(0,82 \%)$ & $3(1,00 \%)$ \\
\hline Otros debates & $1(0,20 \%)$ & $5(1,65 \%)$ & $2(0,54 \%)$ & $5(1,65 \%)$ \\
\hline Análisis y opinión & $37(7,53 \%)$ & $35(11,59 \%)$ & $66(17,99 \%)$ & $38(12,58 \%)$ \\
\hline Entrevistas & $24(4,90 \%)$ & $8(2,65 \%)$ & $19(5,18 \%)$ & $6(1,99 \%)$ \\
\hline \multicolumn{5}{|l|}{ Elecciones 10N } \\
\hline Encuestas & $19(3,88 \%)$ & $3(1,00 \%)$ & $12(3,27 \%)$ & $6(1,99 \%)$ \\
\hline Voto por correo & $6(1,22 \%)$ & $1(0,33 \%)$ & $4(1,09 \%)$ & $1(0,33 \%)$ \\
\hline Conflicto Cataluña & $15(3,05 \%)$ & $10(3,31 \%)$ & $8(2,18 \%)$ & $8(2,65 \%)$ \\
\hline Otros & $2(0,41 \%)$ & - & $1(0,27 \%)$ & $4(1,32 \%)$ \\
\hline Pactos-acuerdos & $6(1,22 \%)$ & $13(4,30 \%)$ & $4(1,09 \%)$ & $11(3,64 \%)$ \\
\hline Generalidades & $46(9,37 \%)$ & $33(10,93 \%)$ & $39(10,63 \%)$ & $35(11,59 \%)$ \\
\hline Total: & 491 (100\%) & $302(100 \%)$ & 367 (100\%) & $302(100 \%)$ \\
\hline
\end{tabular}




\subsection{Posicionamiento}

Los resultados del análisis evidencian que aunque la mayoría de los tweets presentan un tono neutro, se halla una cierta polarización de los contenidos marcada por la línea editorial del medio. Los tweets encuadrados en los macrotemas de "polémicas", "debates", "análisis y opinión" y "actos de campaña" son en los que se aprecia en un mayor grado una intención de fortalecer o debilitar a algún líder político o partido. Este propósito se infiere por una parte por la propia temática con la que aparece vinculado el candidato, o por otra, por la narrativa o elemento gráfico del tweet. En cuanto a los mensajes en los que se posicionan las cabeceras -aunque, como se ha comentado anteriormente, predominan los de carácter neutro-, destacan los tweets negativos, lo cual confronta con el corto peso cuantitativo que presentan los mensajes positivos.

Si bien a la hora de posicionarse se aprecia una clara correlación con la línea ideológica del diario, esta tendencia queda demostrada de manera cuantitativa en la tabla 5. La mayoría de los mensajes negativos de El país - de corte progresista- son referidos a Vox y en El mundo y $A B C$-de tendencia conservadora- al PSOE. La vanguardia históricamente ha tenido un posicionamiento moderado, e intenta mantener un equilibrio entre independistas y constitucionalistas. De ahí la razón por la que no se registra ningún tweet negativo sobre Unidas Podemos y algunos de los mensajes mediante los que pretende menoscabar a otros partidos son sobre sucesos vinculados a la causa independentista.

\section{Aunque la mayoría de los tweets presen- tan un tono neutro, se halla una cierta polarización de los contenidos marcada por la línea editorial}

Las líneas editoriales de los diarios se manifiestan predominantemente en la reiterada publicación de mensajes en los que se exponen al votante informaciones con las que se pretende deteriorar la imagen del partido (trama Gürtel, caso ERE de Andalucía, inspección a las empresas del hermano de Ximo Puig -PSOE-, la firma de proyectos por parte de Rocío Monasterio -Vox- sin tener la titulación, o el veto de Vox a los periodistas de Prisa) más que mensajes en los que muestran un posicionamiento claro a favor de un partido. Por tanto se constata que en los tweets en los que se manifiesta una postura definida las cabeceras optan principalmente por mensajes negativos con el propósito de debilitar a determinados partidos, en lugar de tweets en los que se destaque la labor o ideario de otros. Es preciso señalar que, además de los principales cinco partidos ( $P S O E, P P$, Ciudadanos, Unidas Podemos y Vox), en menor medida también se aborda la actualidad y la campaña de otros candidatos. En este rango, Más País, PNV y ERC son los partidos con mayor incidencia en la cobertura de la campaña electoral.

Tabla 5. Tono empleado por el medio para referirse a los partidos

\begin{tabular}{|c|c|c|c|c|c|}
\hline Tono & Partido & Elpaís & El mundo & $A B C$ & La vanguardia \\
\hline \multirow{6}{*}{ Positivo } & PSOE & $4(0,81 \%)$ & - & - & - \\
\hline & $P P$ & $1(0,20 \%)$ & $2(0,66 \%)$ & $1(0,27 \%)$ & - \\
\hline & $C^{\prime} s$ & - & - & - & - \\
\hline & $U P$ & $1(0,20 \%)$ & $2(0,66 \%)$ & - & - \\
\hline & Vox & $2(0,41 \%)$ & $1(0,33 \%)$ & - & $1(0,33 \%)$ \\
\hline & Otros & - & $1(0,33 \%)$ & - & - \\
\hline \multirow{6}{*}{ Negativo } & PSOE & $9(1,83 \%)$ & $31(10,26 \%)$ & $36(9,81 \%)$ & $10(3,31 \%)$ \\
\hline & $P P$ & $14(2,85 \%)$ & $1(0,33 \%)$ & $1(0,27 \%)$ & $2(0,66 \%)$ \\
\hline & $C^{\prime} s$ & $13(2,64 \%)$ & $2(0,66 \%)$ & $4(1,09 \%)$ & $2(0,66 \%)$ \\
\hline & $U P$ & $1(0,20 \%)$ & $6(1,99 \%)$ & $3(0,82 \%)$ & - \\
\hline & Vox & $36(7,33 \%)$ & $3(0,99 \%)$ & $8(2,18 \%)$ & $8(2,65 \%)$ \\
\hline & Otros & $3(0,61 \%)$ & $3(0,99 \%)$ & $5(1,36 \%)$ & $1(0,33 \%)$ \\
\hline \multirow{6}{*}{ Neutro } & PSOE & $62(12,63 \%)$ & $41(13,58 \%)$ & $34(9,26 \%)$ & $47(15,56 \%)$ \\
\hline & $P P$ & $51(10,39 \%)$ & $48(15,89 \%)$ & $48(13,08 \%)$ & $42(13,91 \%)$ \\
\hline & $C^{\prime} s$ & $52(10,59 \%)$ & $21(6,95 \%)$ & $34(9,26 \%)$ & $18(5,96 \%)$ \\
\hline & $U P$ & $37(7,53 \%)$ & $21(6,95 \%)$ & $22(5,99 \%)$ & $21(6,95 \%)$ \\
\hline & Vox & $25(5,09 \%)$ & $21(6,95 \%)$ & 40 (10,90\%) & $22(7,28 \%)$ \\
\hline & Otros & $19(3,87 \%)$ & $18(5,96 \%)$ & $20(5,45 \%)$ & $29(9,60 \%)$ \\
\hline \multicolumn{2}{|c|}{ Sin especificar partido } & $195(39,71 \%)$ & $99(32,78 \%)$ & $129(35,15 \%)$ & 109 (36,09\%) \\
\hline
\end{tabular}

$(*)$ En varios tweets se hace referencia a más de un partido.

\section{Discusión y conclusiones}

Se constata el notable interés que despiertan los procesos electorales en los medios de comunicación, registrándose una elevada publicación de mensajes relativos a esta cuestión en Twitter durante la campaña electoral. En referencia a los propósitos planteados, como se pretendía en el $\mathrm{O} 1$, se aprecia que este asunto tiene una gran incidencia en la agenda y cobertura realizada, refiriéndose de media a la campaña electoral, al menos, uno de cada cinco tweets, llegándose 
a alcanzar valores superiores al 30\% en las jornadas de mayor quehacer político. La actividad y cobertura realizada es abundante y sostenida en los cuatro casos, aunque El país es el medio en el que tiene más incidencia la campaña electoral. La monitorización de los tweets ha permitido establecer un patrón o rasgo de actuación: el día de la celebración y el posterior al debate electoral junto al cierre de campaña son las jornadas en las que se concentran la mayoría de las publicaciones, cuyo número desciende considerablemente en los días de fines de semana.

También se persigue identificar los rasgos definitorios de los mensajes, así como la interacción lograda (O2). A excepción de la variable "tipo" -en la que predominan los tweets básicos-y de la abundante inclusión de enlaces dirigidos al propio medio, se aprecian divergencias en cuanto a la construcción del mensaje de los cuatro medios, aunque sí que se pueden establecer similitudes entre cabeceras en diversas variables. El país y La vanguardia publican mensajes más elaborados que $E I$ mundo y $A B C$, que se limitan en una elevada proporción a reproducir el titular de la noticia. En cuanto al empleo de elementos visuales y hashtags, la cabecera de Prisa destaca en este aspecto, registrándose un uso muy similar entre los otros medios, aunque no abundan estos recursos en ninguno de los medios estudiados. Esta exigua presencia de recursos visuales y etiquetas podría considerarse un desaprovechamiento de las posibilidades que suministra Twitter para, por una parte, presentar los contenidos de manera más visual y adaptados a la nueva realidad comunicativa planteada por Renó y Barcellos (2017) -quienes ponen en valor la imagen en detrimento del texto-y, por otra, la creación de conversaciones en torno a los mensajes del medio. Pese a la naturaleza dialógica de esta red social, no se detectan mensajes conversacionales con los lectores. Esto último apuntala los hallazgos de investigaciones sobre la prensa española e internacional en Twitter (Domingo et al., 2008; González-Molina; Ramos-del-Cano, 2014).

En líneas generales no se aprovechan las posibilidades dialógicas y técnicas que proporciona Twitter, lo que dificulta la creación de comunidad entre los lectores

De acuerdo con los resultados, los medios que mejor engagement logran son El país y El mundo. No existen similitudes en estos dos diarios en ninguno de los parámetros estudiados relativos a la estructura o aspectos formales del mensaje, aunque cabe precisar que, dentro del discreto uso que hacen todos los rotativos de los elementos gráficos, en estas dos cabeceras tiene mayor incidencia. Los otros factores que podrían propiciar un mayor engagement serían la temática y los protagonistas del tweet. A partir del análisis se identifican los aspectos con mayor potencial de viralización o reacción en la audiencia:

- las polémicas sobre candidatos o partidos y el debate electoral del 4N -en cuanto a temáticas-;

- los mensajes protagonizados por Unidas Podemos y Vox y/o sus respectivos candidatos, Pablo Iglesias y Santiago Abascal.

En todo ello destacan los diarios de Prisa y Unidad Editorial.

Otro rasgo común que se detecta en los cuatro perfiles estudiados es que el público tiende principalmente a señalar like o favorito más que a compartir los contenidos (retweets), un patrón de actuación que viene a corroborar lo señalado por Fernández-Gómez y Martín-Quevedo (2018).

En respuesta a 03 , se detecta que la cobertura de los diarios gravita en torno a dos aspectos predominantes:

- los debates electorales;

- los actos de campaña.

En menor medida, las piezas sobre análisis y opinión y las noticias negativas sobre algún partido político o candidato tienen también una notable incidencia, apreciándose en estos dos últimos macrotemas mayores evidencias de la polarización política que caracteriza al sistema de medios español. En lo referido al tono del mensaje (O4), el análisis ha puesto de manifiesto que mayoritariamente los tweets presentan un tono neutro, aunque, en menor medida, también se registran diversas piezas en las que se pretende fortalecer o debilitar la opinión pública sobre algún candidato o partido. En estos casos sí hay correspondencia entre la línea editorial del medio y el tono del mensaje. Se cuantifica una cifra considerable de tweets negativos sobre el PSOE en El mundo y $A B C$ y sobre Vox en El país. En La vanguardia se aprecia una ausencia de mensajes desfavorables sobre Unidas Podemos. Muchos de los contenidos negativos salen a la luz de manera estratégica en el período electoral pese a no propiciarse el acontecimiento en ese mismo momento y otros se basan en la recuperación con alguna leve variación de hechos anteriores. Todos ellos tenían el claro propósito de influir en el electorado, ejerciendo la crispación según la afinidad a un partido determinado. Se verifica el escenario mediático trazado por Teruel-Rodríguez (2013, p. 189), quien alerta sobre el "paralelismo partidista" de las cabeceras españolas. Para lograr su finalidad se observa que optan por la reiteración de las piezas que tienen un mayor valor persuasivo, repitiendo a lo largo de los días varios tweets. Existe por tanto un esfuerzo de los medios dirigido a conseguir la asimilación de determinados mensajes, evidenciándose ello en una mayor frecuencia de publicación.

A modo de conclusión, se aprecia que todos los diarios analizados llevan a cabo una extensa cobertura -en términos cuantitativos- de la campaña electoral en Twitter, lo que lleva a considerar que durante los períodos electorales la agenda mediática gira en torno a este hecho, reflejándose así la relevancia del mismo. La utilización de Twitter como plataforma para la difusión de contenidos refuerza lo expuesto en otras investigaciones en las que se destaca la función diseminadora de esta red social (García-De-Torres et al., 2011; Alonso-Muñoz; Miquel-Segarra; Casero-Ripollés, 2016). Sin embargo, la moderada originalidad de los asuntos abordados y el discreto empleo de elementos gráficos 
y etiquetas o hashtags durante la cobertura denota un cierto conservadurismo o inmovilismo que repercute negativamente en la explotación del hecho noticioso -las elecciones y la campaña electoral- y la propia red social, no aprovechando las posibilidades técnicas y dialógicas que proporciona Twitter para crear comunidad entre sus lectores. Por ello, quedaría para otras investigaciones explorar si se mantienen estos mismos hábitos en posteriores procesos electorales en la prensa española o un análisis comparativo en el que se abordase el empleo que hacen de las redes sociales las cabeceras extranjeras de referencia durante similares períodos.

\section{Referencias}

Alonso-Muñoz, Laura; Miquel-Segarra, Susana; Casero-Ripollés, Andreu (2016). “Un potencial comunicativo desaprovechado. Twitter como mecanismo generador de diálogo en campaña electoral”. Obra digital, n. 11, pp. 39-58. https://www.raco.cat/index.php/ObraDigital/article/view/315496/405597

Artwick, Claudette G. (2013). "Reporters on Twitter. Product or service?". Digital journalism, v. 1, n. 2, pp. $212-228$. https://doi.org/10.1080/21670811.2012.744555

Barredo, Daniel (2015). “El análisis de contenido. Una introducción a la cuantificación de la realidad”. Revista San Gregorio, n. 1, pp. 26-31.

http://revista.sangregorio.edu.ec/index.php/REVISTASANGREGORIO/article/view/113

https://doi.org/10.36097/rsan.v0i0.113

Bruns, Axel; Burgess, Jean (2012). "Researching news discusion on Twitter: New methodologies". Journalism studies, v. 13, n. 5-6, pp. 1-13.

https://doi.org/10.1080/1461670X.2012.664428

Castillo-Esparcia, Antonio; García-Ponce, Damián; Smolak-Lozano, Emilia (2013). “Movimientos sociales y estrategias de comunicación. El caso del 15-M y de Occupy Wall Street". Estudios sobre el mensaje periodístico, v. 19, n. 1, pp. 71-89. https://doi.org/10.5209/rev_ESMP.2013.v19.n1.42508

Chung, Jin-Young; Buhalis, Dimitrios (2008). "Information needs in online social networks". Information technology \& tourism, v. 10, n. 4, pp. 267-281.

https://www.academia.edu/181213/Chung_Jin_Young_and_Buhalis_D._2008_Information_needs_in_online_social_ networks_Information_Technology_and_Tourism_Vol.10_4_pp.267-282

https://doi.org/10.3727/109830508788403123

Cobos, Tania-Lucía (2015). “Radiografía al comportamiento de la prensa colombiana en Twitter: Casos El heraldo, El universal y El informador". Encuentros, v. 13, n. 1, pp. 85-99.

https://doi.org/10.15665/re.v13i1.351

Criado, José-Ignacio; Martínez-Fuentes, Guadalupe; Silván, Aitor (2013). “Twitter en campaña: las elecciones municipales españolas de 2011". Revista de investigaciones políticas y sociológicas, v. 12, n. 1, pp. 93-113.

https://revistas.usc.gal/index.php/rips/article/view/1307

De-Aguilera, Miguel; Casero-Ripollés, Andreu (2018). “¿Tecnologías para la transformación? Los medios sociales ante el cambio político social". Icono14, v. 16, n. 1, pp. 1-21.

https://doi.org/10.7195/ri14.v16i1.1162

Domingo, David; Masip, Pere; Costera-Meijer, Irene (2014). "Tracing news digital networks. Towards an integrated framework of the dynamics of news production, circulation and use". Digital journalism, v. 3, n. 1, pp. 53-67.

https://doi.org/10.1080/21670811.2014.927996

Domingo, David; Quandt, Thorsten; Heinonen, Ari; Paulussen, Steve; Singer, Jane B.; Vujnovic, Marina (2008). “Participatory journalism practices in the media and beyond: An international comparative study of initiatives in online newspapers". Journalism practice, v. 2, n. 3, pp. 326-342.

https://doi.org/10.1080/17512780802281065

Enli, Gunn-Sara; Skogerbo, Eli (2013). “Personalized campaigns in party-centred politics. Twitter and Facebook as arenas for political communication". Information, communication \& society, v. 16, n. 5, pp. 757-774.

https://doi.org/10.1080/1369118X.2013.782330

Fernández-Gómez, Erika; Martín-Quevedo, Juan (2018). "La estrategia de engagement de Netflix España en Twitter". El profesional de la información, v. 27, n. 6, pp. 1292-1302.

https://doi.org/10.3145/epi.2018.nov.12 
Gainous, Jason; Wagner, Kevin M. (2014). Tweeting to power. The social media revolution in American Politics. Oxford: Oxford University Press. ISBN: 9780199965076

García-Ortega, Carmela; Zugasti-Azagra, Ricardo (2014). “La campaña virtual en Twitter: análisis de las cuentas de Rajoy y de Rubalcaba en las elecciones generales de 2011”. Historia y comunicación social, v. 19, pp. 299-311.

https://doi.org/10.5209/rev_HICS.2014.v19.45029

García-de-Torres, Elvira; Yezers'Ka, Lyudmyla; Rost, Alejandro; Calderín, Mabel; Edo, Concha; Rojano, Miladys; Said, Elías; Jerónimo, Pedro; Arcila, Carlos; Serrano, Ana; Badillo, Jorge; Corredoira, Loreto (2011). "Uso de Twitter y Facebook por los medios iberoamericanos". El profesional de la información, v. 20, n. 6, pp. 611-620.

https://doi.org/10.3145/epi.2011.nov.02

Gómez-Calderón, Bernardo; Roses, Sergio; Paniagua-Rojano, Francisco-Javier (2017). “La campaña en 140 caracteres. Empleo de Twitter por parte de los candidatos de los partidos mayoritarios ante las elecciones generales de 2016 en España". El profesional de la información, v. 26, n. 5, pp. 816-823.

https://doi.org/10.3145/epi.2017.sep.04

González-Molina, Sonia; Ramos-del-Cano, Fátima (2014). “Las redes sociales en el ámbito periodístico: ¿cómo usan los medios europeos de referencia sus perfiles en Twitter y Facebook?". Comunicación y hombre, n. 10, pp. 37-52.

https://comunicacionyhombre.com/article/las-redes-sociales-ambito-periodistico-usan-los-medios-europeosreferencia-perfiles-twitter-facebook/1234

Guemureman, Silvia; Fridman, Denise; Graziano, Florencia; Jorolinsky, Karen; López, Ana-Laura; Pasin, Julia; Salgado, Vanesa (2011). "Rol de los medios de comunicación en el despliegue de los mecanismos de control social, proactivos y reactivos. Legitimación de la violencia estatal contra los jóvenes pobres y su vinculación discursiva con la 'delincuencia'”. En: VI Jornadas de Sociología de la UNLP, 9-10 de diciembre, Ensenada, Argentina.

http://www.memoria.fahce.unlp.edu.ar/trab_eventos/ev.5699/ev.5699.pdf

Hong, Sounman (2012). "Online news on Twitter: newspaper social media adoption and their online readership". Information, economics and policy, v. 24, n. 1, pp. 69-74.

https://doi.org/10.1016/j.infoecopol.2012.01.004

Huertas, Assumpció; Setó-Pàmies, Dolors; Míguez-González, María-Isabel (2015). “Comunicación de destinos turísticos a través de los medios sociales". El profesional de la información, v. 24, n. 1, pp. 15-21.

https://doi.org/10.3145/epi.2015.ene.02

Igartua-Perosanz, Juan-José (2006). Métodos cuantitativos de investigación en comunicación. Barcelona: Bosch. ISBN: 9788497902717

Ju, Alice; Jeong, Sun-Ho; Chyi, Hsiang-Iris (2014). "Will social media save newspapers? Examining the effectiveness of Facebook and Twitter as news platforms". Journalism practice, v. 8, n. 1, pp. 1-17.

https://doi.org/10.1080/17512786.2013.794022

Krippendorff, Klaus (1990). Metodología de análisis de contenido. Teoría y práctica. Barcelona: Paidós. ISBN: 8475096271

Linares-Lanzman, Juan; Pérez-Altable, Laura (2015). “Usos de Twitter durante el 15M. El caso de la prensa catalana”. Sur le journalisme, v. 4, n. 1, pp. 64-75.

http://www.surlejournalisme.kinghost.net/rev/index.php/slj/article/view/199

López-Meri, Amparo (2015). “Redes sociales y campañas electorales: Twitter como fuente informativa en las elecciones catalanas del \#25N". Comunicació, v. 32, n. 2, pp. 115-137.

https://doi.org/10.2436/20.3008.01.138

Maciá, Fernando; Santonja, María (dir.) (2016). Marketing en redes sociales. Madrid: Anaya Multimedia. ISBN: 97884 41537262

Micó, Josep-Lluís; Casero-Ripollés, Andreu (2014). "Political activism online: organization and media relations in the case of 15M in Spain". Information, communication \& society, v. 17, n. 7, pp. 858-871.

https://doi.org/10.1080/1369118X.2013.830634

Moreno, Manuel (2019). "Twitter tiene 12 veces menos usuarios diarios que Facebook". Trecebits, 29 julio. https://www.trecebits.com/2019/07/29/twitter-usuarios-diarios

Nadal-Martínez, Ricardo (2013). "Twitter y prensa tradicional. Muestra de la cobertura del debate del estado de la nación de 2013". Fòrum de recerca, n. 18, pp. 543-558.

https://doi.org/10.6035/ForumRecerca.2013.37

Palomo, Bella; Palau-Sampio, Dolors (2016). "El periodista adaptativo. Consultores y directores de innovación analizan las cualidades del profesional de la comunicación”. El profesional de la información, v. 25, n. 2, pp. 188-195.

https://doi.org/10.3145/epi.2016.mar.05 
Paz-García, Ana-Pamela; Spinosa, María-Mercedes (2014). “Periodismo político en Twitter: la cobertura electoral en \#CBAVota (Argentina, 2011)". Cuadernos.info, n. 34, pp. 133-152.

https://doi.org/10.7764/cdi.34.558

Pew Research Center (2018). Actitudes con respecto a los medios informativos y a la política española.

https://www.pewresearch.org/global/wp-content/uploads/sites/2/2018/11/PJ_2018-05-17_Media-Politics-WesternEurope_Fact-Sheet_Spain-in-Spanish1.pdf

Pujol, Francesc (2019). “La prensa en Twitter: análisis de actividad e impacto en 2018". Cuadernos de periodistas, n. 38, pp. 54-67.

http://www.cuadernosdeperiodistas.com/media/2019/10/Francesc-puyol_compressed.pdf

Quevedo-Redondo, Raquel; Portalés-Oliva, Marta; Berrocal-Gonzalo, Salomé (2016). “El uso de la imagen en Twitter durante la campaña electoral municipal de 2015 en España”. Revista latina de comunicación social, n. 71, pp. 85-107. https://doi.org/10.4185/RLCS-2016-1085

Renó, Denis; Barcellos, Jefferson (2017). "Paradoxos da fotografia na nova ecologia dos meios: Inovação tecnológica?". Teknokultura, v. 14, n. 2, pp. 363-371. https://doi.org/10.5209/TEKN.56439

Rodríguez-Andrés, Roberto; Ureña-Uceda, Daniel (2011). “Diez razones para el uso de Twitter como herramienta en la comunicación política y electoral”. Comunicación y pluralismo, v. 10, pp. 89-106.

https://core.ac.uk/download/pdf/50605323.pdf

Said-Hung, Elías; Arcila-Calderón, Carlos (2011). “Los líderes de opinión en Colombia, Venezuela e Irán. El caso de los 20 usuarios más vistos en Twitter". Comunicación y sociedad, v. 24, n. 1, pp. 75-100.

https://revistas.unav.edu/index.php/communication-and-society/article/view/36225

Salgado, Carmen; Zamarra-López, María-Mercedes (2013). "Twitter dinamiza las sinergias entre las redes sociales y el periodismo". En: Sabés-Turmo, Fernando; Verón-Lassa, José-Juan (coords.). Comunicación y la red. Nuevas formas de periodismo. Zaragoza: Asociación de Periodistas de Aragón. ISBN: 9788487175497

Sánchez-García, Pilar; Campos-Domínguez, Eva; Berrocal-Gonzalo, Salomé (2015). “Las funciones inalterables del periodista ante los perfiles multimedia emergentes". Revista latina de comunicación social, n. 70, pp. 187-208.

https://doi.org/10.4185/RLCS-2015-1042

Tandoc Jr., Edson C.; Vos, Tim P. (2016). "The journalist is marketing the news: Social media in the gatekeeping process". Journalism practice, v. 10, n. 8, pp. 950-966.

https://doi.org/10.1080/17512786.2015.1087811

Teruel-Rodríguez, Laura (2013). "El posicionamiento editorial de la prensa española sobre la polarización política". En: Sanders, Karen; Canel, María-José; Capdevila, Arantxa; Gurrionero, Mario. Estudios de comunicación política: libro del año 2012. Madrid: Editorial Tecnos. ISBN: 9788430955305

https://www.researchgate.net/publication/269629319_El_posicionamiento_editorial_de_la_prensa_espanola_sobre_ la_polarizacion_politica

Tumasjan, Andranik; Sprenger, Timm O.; Sandner, Philipp G.; Welpe, Isabell M. (2010). “Predicting elections with Twitter: What 140 characters reveal about political sentiment". In: International AAAl conference on weblogs and social media. Washington DC: George Washington University. https://www.aaai.org/ocs/index.php/ICWSM/ICWSM10/paper/view/1441

Túñez-López, Miguel (2012). "Los periódicos en las redes sociales: audiencias, contenidos, interactividad y estrategias comerciales". Estudios sobre el mensaje periodístico, v. 18, n. 1.

https://doi.org/10.5209/rev_ESMP.2012.v18.n1.39367

Wimmer, Roger D.; Dominick, Joseph R. (1996). La investigación científica de los medios de comunicación. Una introducción a sus métodos. Barcelona: Bosch. ISBN: 9788476763599

Zamarra-López, María-Mercedes (2015). “Análisis de los diarios digitales españoles más influyentes y su presencia en las redes sociales". Estudios sobre el mensaje periodístico, v. 21, pp. 155-170.

https://doi.org/10.5209/rev_ESMP.2015.v21.51137 